Hydroécol. Appl. (1989) 1/2, pp. 19-31

\title{
Modélisation du régime thermique sur le littoral nord breton
}

\author{
Joëlle Riou
}

Electricité de France. Direction des Etudes et Recherches 6, quai Watier 78400 Chatou France

Résumé - Un modèle thermique bidimensionnel, à deux couches homogènes d'épaisseurs variables, a été développé sur la bande littorale Roscoff-Saint Malo (Bretagne nord, France). Sont pris en compte les échanges d'énergie à l'interface air-mer, la turbulence induite par le vent et les courants, les phénomènes advectifs et diffusifs liés au vent et à la marée. Les résultats ont été validés en plusieurs stations; le modèle décrit correctement le régime thermique côtier et permet notamment de reproduire des chroniques interannuelles de température sur plusieurs décennies.

Summary - $A$ two dimensionnal numerical model, having two homogeneous layers, has been developped to assess the coastal temperature from Roscoff to Saint Malo (North Britain, France). It includes energy transfer at the air-sea interface, turbulence induced by wind and currents, advection and diffusion due to wind and tides. Results have been validated over several stations. This model leads to a good description of the thermal distribution in the coastal zone and it is able to reproduce temperatures series over several decades in time.

\section{Introduction}

Si les études écologiques sur le domaine de la Manche se sont souvent limitées aux côtes anglaises et aux régions frontales, la côte Nord-Bretonne reste un terrain encore peu exploité, tant par les moyens expérimentaux qu'informatiques.

Dans le prolongement du travail effectué sur la Manche à une échelle assez large par Agoumi en 1985 [1], un programme d'études expérimentales et de modélisation a débuté en 1987, avec pour objectif l'appréhension de l'écosystème phytoplanctonique sur la bande littorale Roscoft-Saint Malo.

Nous nous proposons, dans cette note, de présenter la partie thermique du modèle : après avoir rappelé sa structure, nous examinerons les en- 
seignements qu'il peut apporter concernant le régime thermique des eaux côtières de Bretagne Nord.

\section{Présentation du site : Caractéristiques hydrodynamiques}

La zone géographique retenue est la bande côtière située entre Roscoff à l'ouest et Saint Malo à l'est : elle s'étend sur une trentaine de kilomètres au large (Fig. 1). La côte Nord Bretonne, d'orientation générale ouestest, présente un littoral très entaillé, tant par de profonds mais étroits abers que par de larges baies (baies de Morlaix, Lannion, Saint-Brieuc). La bathymétrie des fonds a un aspect extrêmement tourmenté, lié à la présence de nombreux hauts-fonds et ilôts et les profondeurs décroissent très rapidement de la côte vers le large. L'une des principales caractéristiques de cette région est l'intensité de ses courants de marée, qui peut atteindre 4 nœuds à 3 ou 4 kilomètres des côtes : ils sont de type alternatif, le flot portant de manière générale à l'est, et le jusant à l'ouest [3] [4]. Les marnages associés sont eux aussi très élevés (10 mètres à Saint-Malo, 7,3 mètres à Morlaix). Les vents dominants sont de secteur SW à NW et on note une prépondérance des coups de vent (supérieurs à $45 \mathrm{~km} / \mathrm{h}$ à 10 mètres du sol) de secteur Ouest.

\section{Rappel du modèle thermique : mécanismes et équations}

\section{a) Principe du modèle :}

Nous avons repris le schéma bidimensionnel horizontal, à deux

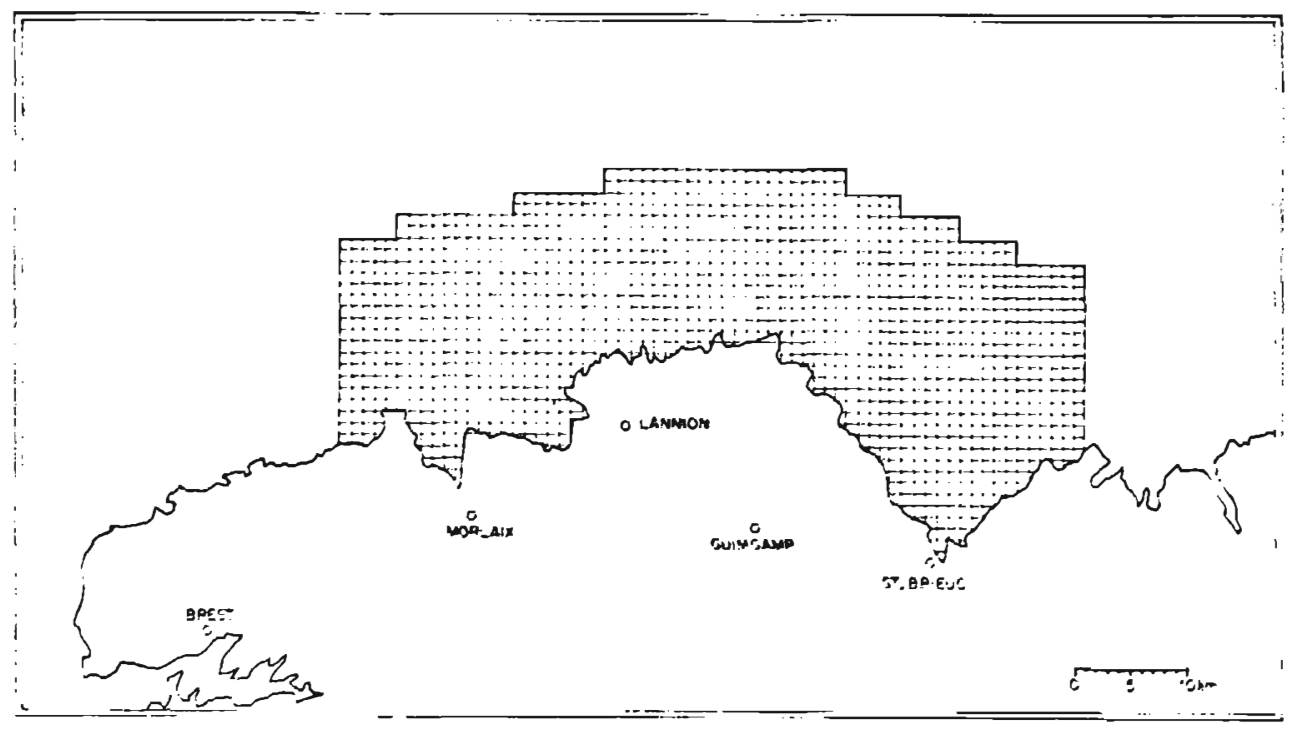

Fig. 1. Côte Nord bretonne, emprise du modèle et maillage. 
couches homogènes d'épaisseurs variables, développé sur l'ensemble de la Manche par Agoumi (1982) [2]. Le modèle a été adapté pour s'appliquer à un maillage plus fin (mailles de $2 \mathrm{~km}$ de côté au lieu de $10 \mathrm{~km}$, soit un nombre total de 2145 mailles) (voir Figure 1).

Les principaux mécanismes déterminant l'évolution thermique des masses d'eau sont pris en compte, à savoir :

- les échanges d'énergie à l'interface air-mer,

- la turbulence induite par le vent en surface et par les courants de marée au fond (affectant la structure verticale),

- les phénomènes de transport advectifs et diffusifs, dus aux courant de marée et au vent.

Tous ces mécanismes sont récapitulés sur la figure 2 .

\section{Dans notre approche :}

a) Les masses d'eau en surface sont soumises à des conditions météorologiques variables. Ces échanges énergétiques sont un facteur essentiel pour la représentation du régime thermique. Ils se traduisent par des fonctions classiques de flux de chaleur (Klein, 1980) [5] qui sont évaluées à partir de données trihoraires des paramètres météorologiques :

- vitesse du vent

- température de l'air

- humidité relative de l'air

- pression atmosphérique

- nébulosité

b) L'existence d'une stratification thermique des masses d'eau dépend de l'action du vent en surface et des courants de marée au fond : l'énergie cinétique turbulente créée par chacun de ces facteurs va tendre à entraîner les masses d'eau d'une couche à l'autre et modifier ainsi la position de la discontinuité thermique. Ce mécanisme est reproduit dans le modèle par l'intermédiaire de vitesses d'entraînement, We1 et We2 $(\mathrm{m} / \mathrm{s})$, estimées sur la base des énergies cinétiques turbulentes produites à la surface et au fond. Cette représentation des échanges entre couches est celle utilisée dans les modèles dits "intégraux" dont une synthèse a été développée par Niiler et Kraus en 1977 [6].

c) En ce qui concerne les transports horizontaux, nous distinguons deux phénomènes à échelle de temps différente :

- les phénomènes à macro échelle constitués par la circulation résiduelle (circulation issue du filtrage des courants de marée et des courants dus au vent sur des temps de lordre de plusieurs périodes de marée). Ce transport résiduel des masses d'eau se traduit dans le modèle par des termes advectifs, représentés par des vitesses Uc, Vc supposées constantes sur la verticale.

- les phénomènes à méso échelle induits par les courants de marée. Ils sont associés aux échanges dispersifs et schématisés par des coefficients de dispersion différents selon les axes Nord-Sud et Est-Ouest; Kx, Ky sont supposés constants sur l'ensemble du domaine étudié. 

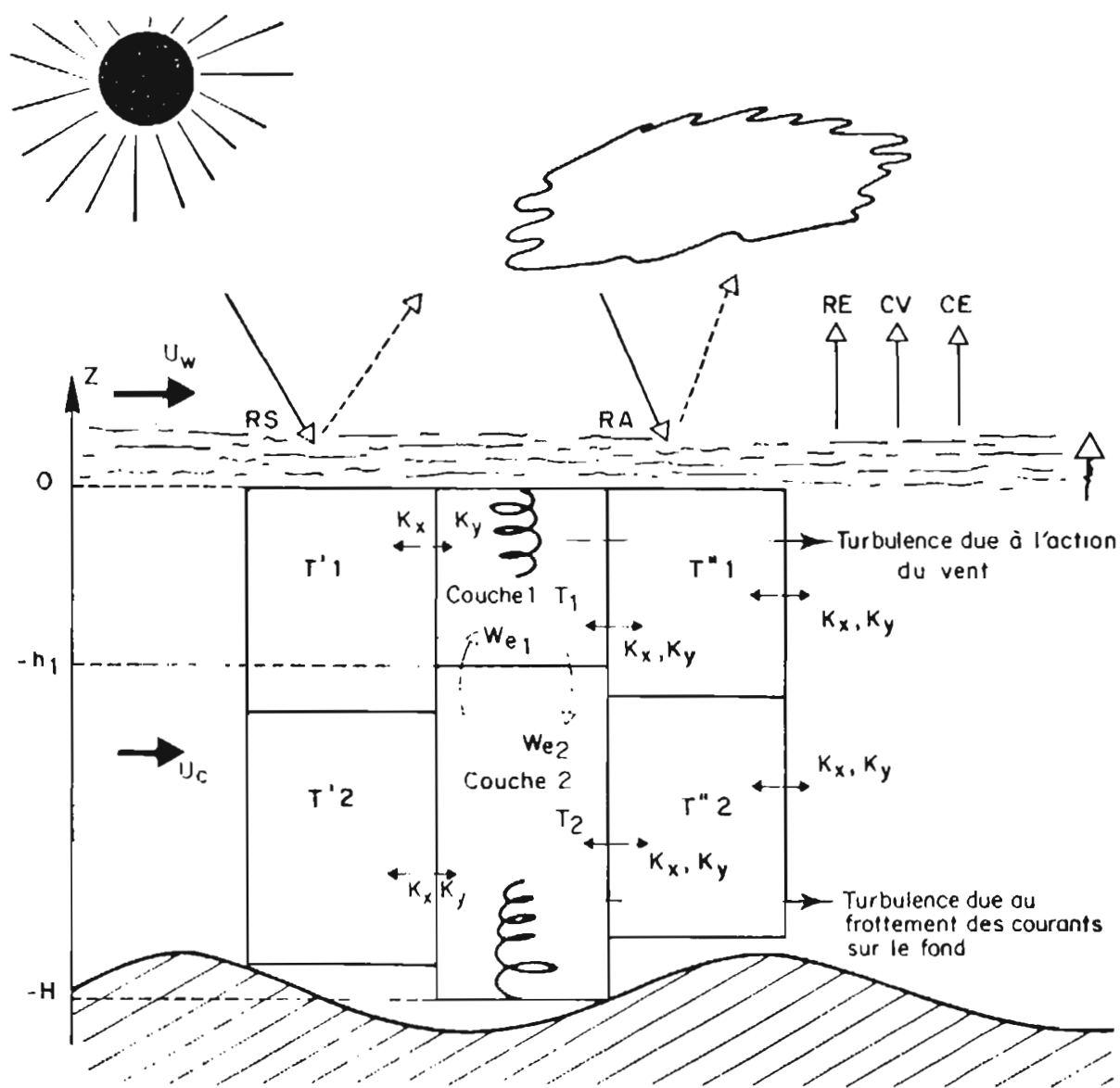

$W_{e_{1}}$. Vitesse d'entrainement des mosses d'eau de la couche 2 à lo couche 1

We2 Vitesse d'entranement des masses d'eou de la couche 1 à lo couche 2.

Uc: Vilesse des mosses d'eau

$U_{w}$. Vitesse du vent

$K_{x}, K_{y}$. Coefficients de dispersion

CE Flux de chaleur dù ò l'évaporation

CV. Flux de chaleur dù à la convection

RE - Rayonnement de I'eau

RS : Royonnement solare

RA Rayonnement atmosphérique

Fig. 2 Schématisation des mécanismes. 


\section{b) Equations du modèle :}

La variation de l'épaisseur de la couche de surface $h_{1}$ dans le temps s'écrit :

$$
\frac{d h_{1}}{d t}=W e_{1}-W e_{2}
$$

où We $\mathrm{H}_{1}$ est la vitesse d'entraînement de la couche inférieure vers la couche supérieure sous l'influence des échanges turbulents générés par le fond et We2 la vitesse d'entraînement de la couche supérieure vers la couche inférieure sous l'influence du vent.

Les variations de température de la couche supérieure (T1) et de la couche inférieure (T2) sont déterminées par l'intégration de l'équation de l'enthalpie sur chacune des deux épaisseurs, en négligeant les effets de diffusion à travers l'interface entre les couches, par rapport aux processus de mélange associés à l'entraînement d'une couche à l'autre. Elles s'écrivent :

$$
\begin{gathered}
\frac{\partial T 1}{\partial t}+U_{c} \frac{\partial T 1}{\partial x}+V_{c} \frac{\partial T 1}{\partial y}=\frac{W e_{1}}{h_{1}}(T 2-T 1) \\
+\frac{-P+R(-h 1)-R(O)}{\rho 0 C p h 1}+\frac{1}{h 1} \frac{\partial}{\partial x}\left(h 1 K x \frac{\partial T 1}{\partial x}\right) \\
+\frac{1}{h 1} \frac{\partial}{\partial y}\left(h 1 K y \frac{\partial T 1}{\partial y}\right) \\
\frac{\partial T 2}{\partial t}+U_{c} \frac{\partial T 2}{\partial x}+V_{c} \frac{\partial T 2}{\partial y}=\frac{W_{2}}{H-h_{1}}(T 1-T 2) \\
+\frac{R(-H)-R(-h 1)}{\rho 0 C p(H-h 1)}+\frac{1}{(H-h 1)} \frac{\partial}{\partial x}\left((H-h 1) K x \frac{\partial T 2}{\partial x}\right) \\
+\frac{1}{(H-h 1)} \frac{\partial}{\partial y}\left((H-h 1) K y \frac{\partial T 2}{\partial y}\right)
\end{gathered}
$$

où $P$ est le terme de pertes énergétiques en surface $\left(\mathrm{W} / \mathrm{m}^{2}\right)$

$R(O)$ le rayonnement solaire en surface $\left(\mathrm{W} / \mathrm{m}^{2}\right)$

$\mathrm{R}(-\mathrm{h} 1)$ le rayonnement solaire à la profondeur h1 $\left(\mathrm{W} / \mathrm{m}^{2}\right)$

$\mathrm{R}(-\mathrm{H})$ le rayonnement solaire à la profondeur $\mathrm{H}\left(\mathrm{W} / \mathrm{m}^{2}\right)$

Les vitesses d'entraînement sont obtenues par intégration de l'équation de l'énergie cinétique turbulente sur h1 et $\left(H-h_{1}\right)$ en supposant les flux turbulents horizontaux négligeables devant les flux turbulents verticaux et avec l'hypothèse de quasi-stationnarité de l'énergie cinétique turbulente.

$$
\begin{gathered}
W e_{2}=\left[\frac { 1 } { ( T 1 - T 2 ) } \left[\frac{2 m_{c} U_{c} *^{3}}{g \alpha\left(H-h_{1}\right)}\right.\right. \\
\left.+\frac{R(-H)+R(-h 1)}{\rho \circ C p}-\frac{2}{\rho \circ c_{p}\left(H-h_{1}\right)} \int_{-H}^{h_{1}} R(z) d z\right] \\
W e_{1}=\frac{1}{(T 1-T 2)}\left[\frac{2 m_{v} U_{v} *^{3}}{g \alpha h 1}\right. \\
-(1-n) \frac{P+|P|}{2 \rho \circ C p}+\frac{P+R(o)+R(-h 1)}{\rho \circ C p} \\
\left.\quad-\frac{2}{\rho \circ C p h_{1}} \int_{-h 1}^{\rho} R(z) d z\right\}
\end{gathered}
$$

où Uc* : vitesse de frottement au fond $(\mathrm{m} / \mathrm{s})$ définie telle que

$$
\begin{aligned}
& U c^{*}=U c \sqrt{c d} \\
& U c: \text { vitesse du courant moyen sur } \\
& \text { la profondeur } H
\end{aligned}
$$

Cd : coefficient de frottement au fond $\left(\mathrm{Cd}=2,1 \times 10^{-3}\right)$

Uv* : vitesse de frottement en surface $(\mathrm{m} / \mathrm{s})$ définie :

$$
U v^{*}=U 10 \sqrt{\frac{\rho_{a} C_{10}}{\rho_{0}}}
$$


exprimée en fonction de U10 vitesse du vent à 10 mètres,

C10 : coefficient de frottement

pa : masse volumique de l'air

$\rho o$ : masse volumique de l'eau

$\mathrm{g}$ : accélération due à la gravité

$\mathrm{Cp}$ : capacité calorifique de l'eau

$\alpha$ : coefficient d'expansion thermique de l'eau

$\mathbf{n}$ : paramètre relatif à la convection $(n=0$ [2])

$m_{v}$ : part de l'énergie cinétique née de l'action du vent en surface utilisée pour le mélange $\left(m_{v}=0.5[2]\right)$

$\mathrm{m}_{\mathrm{c}}$ : part de l'énergie cinétique due au frottement des courants de marée au fond servant au mélange $\left(m_{c}=0.07[2]\right)$.

\section{c) Méthode de résolution}

Le système d'équations est résolu par une méthode explicite aux différences finies : le pas d'espace utilisé dans les calculs est constant et égal à $2 \mathrm{~km}$; le pas de temps associé est de 1 heure.

La détermination des conditions aux limites est rendue délicate du fait de l'existence dans notre système de frontières en mer ouverte : cette difficulté est levée en interpolant à chaque pas de temps les températures calculées par le modèle global de la Manche (Agoumi, [2]) sur les mailles limites de notre domaine d'étude. Sur les frontières terrestres, nous retenons l'hypothèse classique d'absence d'échanges thermiques.

En ce qui concerne les conditions initiales, une étude de sensibilité a montré qu'une perturbation sur l'état initial disparaissait au bout de deux années de simulation.

Aussi, afin de s'assurer d'une mise à l'équilibre avec les conditions météorologiques locales, toute simulation commencera deux années avant la date effective de l'étude.

\section{Données nécessaires à la simulation}

Plusieurs types de données sont nécessaires au fonctionnement du modèle : elle sont obtenues par mesures ou par l'utilisation d'autres modèles numériques.

a) La bathymétrie est reconstituée à partir de valeurs relevées sur les cartes de SHOM, à l'aide d'un programme interactif d'interpolation.

b) Les paramètres météorologiques atmosphériques sont ceux relevés à la station de La Hague, qui s'est révélée la mieux adaptée pour le calcul du régime thermique de l'ensemble de la Manche. Un ajustement local du coefficient de vent permet de mieux les décrire sur la zone géographique choisie.

c) Les informations relatives aux courants sont de deux types et toutes accessibles par modélisation : il nous faut, d'une part connaître les courants de marée instantanés afin de déterminer le niveau de turbulence engendré par la friction de ces courants sur le fond, et d'autre part la circulation résiduelle qui assurera dans le modèle le transport des masses d'eau; les modèles utilisés sont les suivants : 
(i) Un code de calcul de courant développé actuellement au Laboratoire National d'Hydraulique (EDF) qui permet, sur un domaine géographique analogue à celui du modèle ici présenté, de simuler une marée de vives eaux (coefficient 95), pendant toute une période de marée, soit $12 \mathrm{~h} 24 \mathrm{mn}$, (Regimbaud, 1987) [8]. II s'agit d'un modèle bidimensionnel horizontal, résolvant les équations de Saint-Venant par une méthode aux éléments finis, avec un pas de temps de $180 \mathrm{~s}$. II suppose l'homogénéité verticale du courant de marée, hypothèse qui se vérifie sur cette bande côtière (Orgby, 1986) [9]. Dans le cas de marée de coefficient différent, l'intensité du champ de courant moyen est obtenue en supposant la linéarité entre les variations de l'amplitude du courant et celles du coefficient de marée. Cette approximation est justifiée par les données du SHOM. Pour les raisons de stabilité numérique, tous les fonds inférieurs à 10 mètres sont ramenés à cette profondeur, mais les résultats de courants ne sont guère affectés qu'en deçà d'un mille de la côte. Les courants calculés sont ensuite moyennés jour par jour et utilisés sous cette forme dans le modèle thermique.

(ii) Un modèle de circulation résiduelle en Manche (Agoumi, 1985) [1] : la distribution des courants obtenue correspond à un flux permanent parcourant la
Manche d'ouest en est, le mouvement étant supposé instationnel. La valeur utilisée $(15,5$ $\times \mathrm{m}^{3} / \mathrm{s}$ ) est celle évaluée sur une année par Prandle (1978) [10] et prend en compte à la fois les effets de la marée, du vent, et de la différence de niveau entre la Manche et la Mer du Nord. Dans le modèle, elle est modulée tous les mois, afin de rendre compte des variations saisonnières.

\section{Calage du modèle}

Lors de l'élaboration d'un modèle, la nécessité d'en limiter l'envergure entraine l'apparition de paramètres dits d'ajustement ou de calage dont la valeur est réglée selon les conditions particulières de l'étude. Leur détermination requiert des données expérimentales auxquelles on compare les résultats du modèle. De plus, si l'on dispose de deux séries indépendantes de mesures, on peut alors procéder à une validation du modèle de la façon suivante : la première série de mesures va servir à la détermination des paramètres à caler; une fois cette étape franchie, en maintenant fixées les valeurs précédemment trouvées, on vérifiera que le modèle s'accorde avec l'autre série de mesures, sans avoir à retoucher au calage précédent. Dans la plupart des cas le manque de données expérimentales limite l'ajustement du modèle à la seule étape de calage. Sont ici à déterminer : 
- les trois paramètres relatifs aux fonctions d'échange à l'interface air-mer (coefficient de vent apparaissant dans les équations déterminant le flux de chaleur dû à la convection et à l'évaporation à partir du vent, coefficient caractéristique de la couverture nuageuse, coefficient relatif à la part de rayonnement solaire réémis par la masse d'eau).

- les coefficients de dispersion $K x$, Ky.

Nous nous sommes appuyés, lors de la phase de calage, sur une base de données constituée par l'Institut Scientifique et Technique des Pêches Maritimes entre 1960 et 1970, (à partir des relevés mensuels de température en surface et au fond) en trois stations en baie de Roscoff (Fig. 3)
[11]. L'ajustement du modèle sur ces données, avec le maillage de $2 \mathrm{~km}$, conduit, pour les coefficients de dispersion, au choix des valeurs de $\mathrm{Kx}=70 \mathrm{~m}^{2} / \mathrm{s}$ et $\mathrm{Ky}=40 \mathrm{~m}^{2} / \mathrm{s}$.

\section{Validation du modèle}

Son ajustement ayant été réalisé sur la décennie 1960-1970, il s'agit d'examiner les possibilités du modèle à reproduire correctement des chroniques de température sur d'autres périodes temporelles et d'autres endroits du domaine. Les valeurs des coefficients de calage ayant été fixées lors de l'étape précédente, nous avons simulé le régime thermique des eaux du littoral Nord-Breton pendant les années 1985 et 1987, car nous y disposions

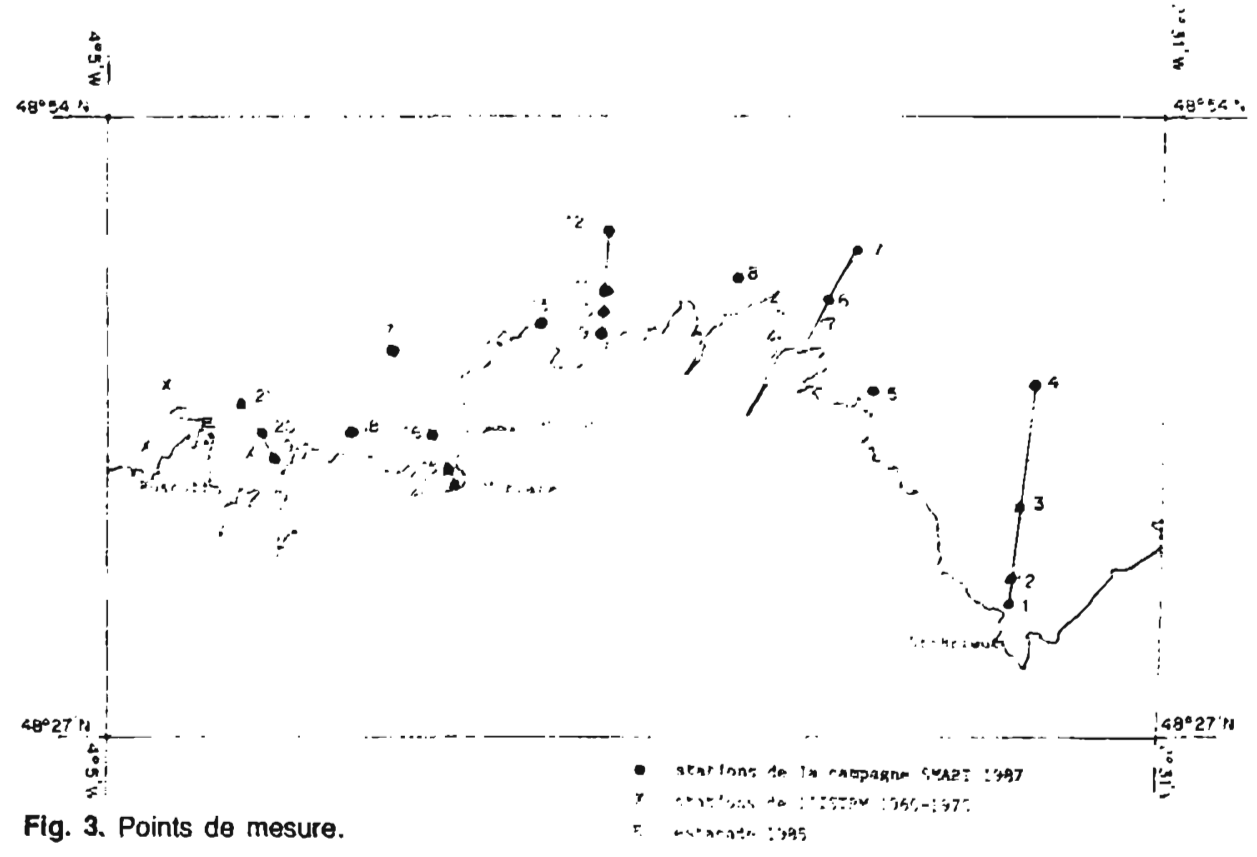


de résultats expérimentaux permettant une confrontation mesures-modèle.

(i) En 1985, un programme interdisciplinaire relatif à l'étude de l'algue Rhizosolenia delicatula a été lancé à Roscoff sous l'impulsion de $\mathrm{Mr}$ Sournia [12] : des suivis journaliers de température ont ainsi été effectués en une station côtière ("estacade") située devant le port de Roscoff (Fig. 3). Le modèle retrace fidèlement cette chronique annuelle, présentant notamment une bonne correspondance des minima et maxima à la fois en amplitude et en phase (Fig. 4). (ii) Dans le cadre de cette étude d'écosystème planctonique sur le littoral Nord-Breton, un programme de mesures a débuté en 1987, l'objectif étant d'acquérir un ensemble de données permettant une couverture spatiale de la zone côtière (Fig. 3). Nous obtenons globalement une bonne cohérence entre cette nouvelle série de températures et les résultats du modèle (Fig. 5), les écarts les plus importants étant observés pour la station la plus côtière (station $n^{\circ} 9$ ), mais ne dépassent pas $0,5^{\circ} \mathrm{C}$.

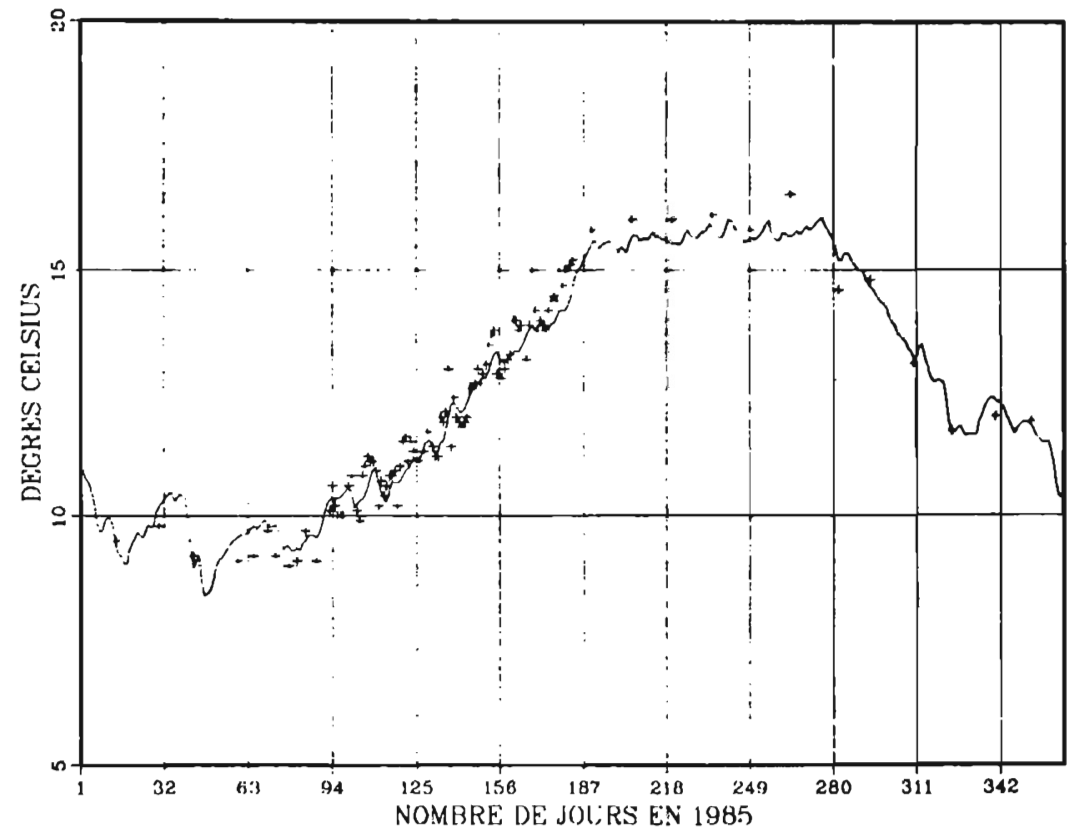

Fig. 4. Résultats du modèle thermique en 1985. Comparaison avec les points de mesure à l'Estacade de Roscotf. 


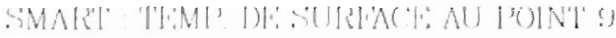

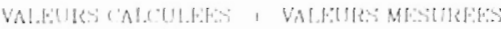

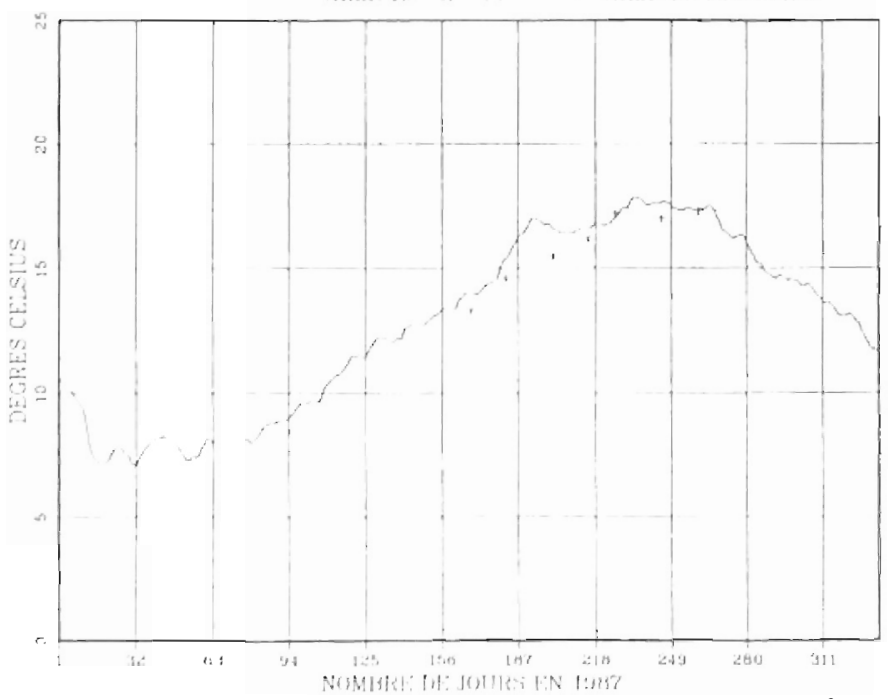

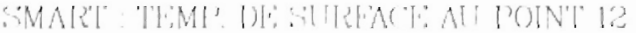

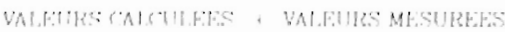

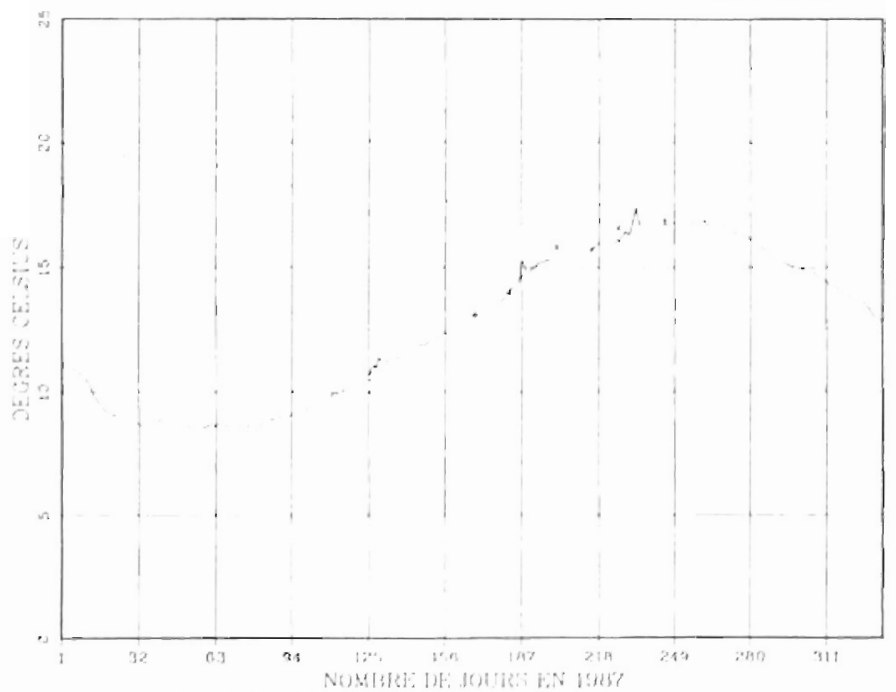

Fig. 5. Résultats du modèle thermique en 1987. Comparaison avec les points de mesure de la campagne SMART. 


\section{Enseignement du modèle et conclusion}

Le modèle côtier donne, comme le montrent les chapitres précédents, une description fidèle du régime thermique au demi-degré près sur l'ensemble du littoral Nord-Breton, à condition d'opter pour l'hypothèse des coefficients de diffusion constants dans le temps et de différencier, pour leur détermination, la baie de SaintBrieuc du reste du domaine.

On notera que ces bons résultats sont obtenus en conservant les mécanismes décrits dans le modèle thermique développé à une échelle plus globale de la Manche [2], ce qui tend à indiquer qu'à l'échelle côtière, les échanges locaux d'énergie à l'interface eau-atmosphère et le mélange vertical et horizontal dans le champ proche sont également les facteurs essentiels gouvernant le régime thermique.

Quelques caractéristiques de la région étudiée peuvent être dégagées par le modèle :

- On met en évidence un net gradient ouest-est, dont le sens varie seIon les saisons; on observe, en hiver, une élévation de température lorsque l'on se déplace d'est en ouest, ce phénomène s'inversant en été (Fig. 6) avec des écarts pouvant atteindre $2,5^{\circ} \mathrm{C}$. Cette particularité est expliquée par la différence d'inertie thermique de masses d'eau, les profondeurs étant plus élevées à l'Ouest qu'à l'Est.

- Les baies de Saint-Brieuc, Lannion et Morlaix constituent des entités particulières; elles présentent des gradients côte-large bien marqués, soumis eux aussi à des variations saisonnières (Fig. 6).

- Les gradients thermiques verticaux ne dépassent pas $0,5^{\circ} \mathrm{C}$ sur la majorité du domaine étudié, et seules les eaux les plus à l'ouest et les plus au large de la zone considérée présentent des écarts pouvant atteindre $1^{\circ} \mathrm{C}$ au mois d'août. Les eaux de la région étudiée sont donc dans l'ensemble, caractéristiques d'une zone bien mélangée verticalement.

Un tel modèle permet en outre de donner une dimension spatiale à l'étude du régime thermique, dimension qui ne pourrait pas être acquise de manière si fine par un travail sur le terrain. II est capable, de plus, de reproduire des chroniques interannuelles de température, ce qui présente un intérêt certain pour toute étude climatologique et écologique (Fig. 7). Ce modèle va désormais servir de support à la représentation de l'écosystème phytoplanctonique sur le littoral Nord-Breton, la température jouant un rôle important sur l'ensemble des variables biologiques.

\section{Références bibliographiques}

[1] Agoumi, A., (1985): Modélisation de l'écosystème pélagique en Manche. Thèse de Docteur ès Sciences, Université Pierre et Marie Curie, Paris 6.

[2] Agoumi, A., (1982) : Modélisation du régime thermique de la Manche. Thèse de Docteur-Ingénieur de l'Ecole Nationale des Ponts et Chaussées. 

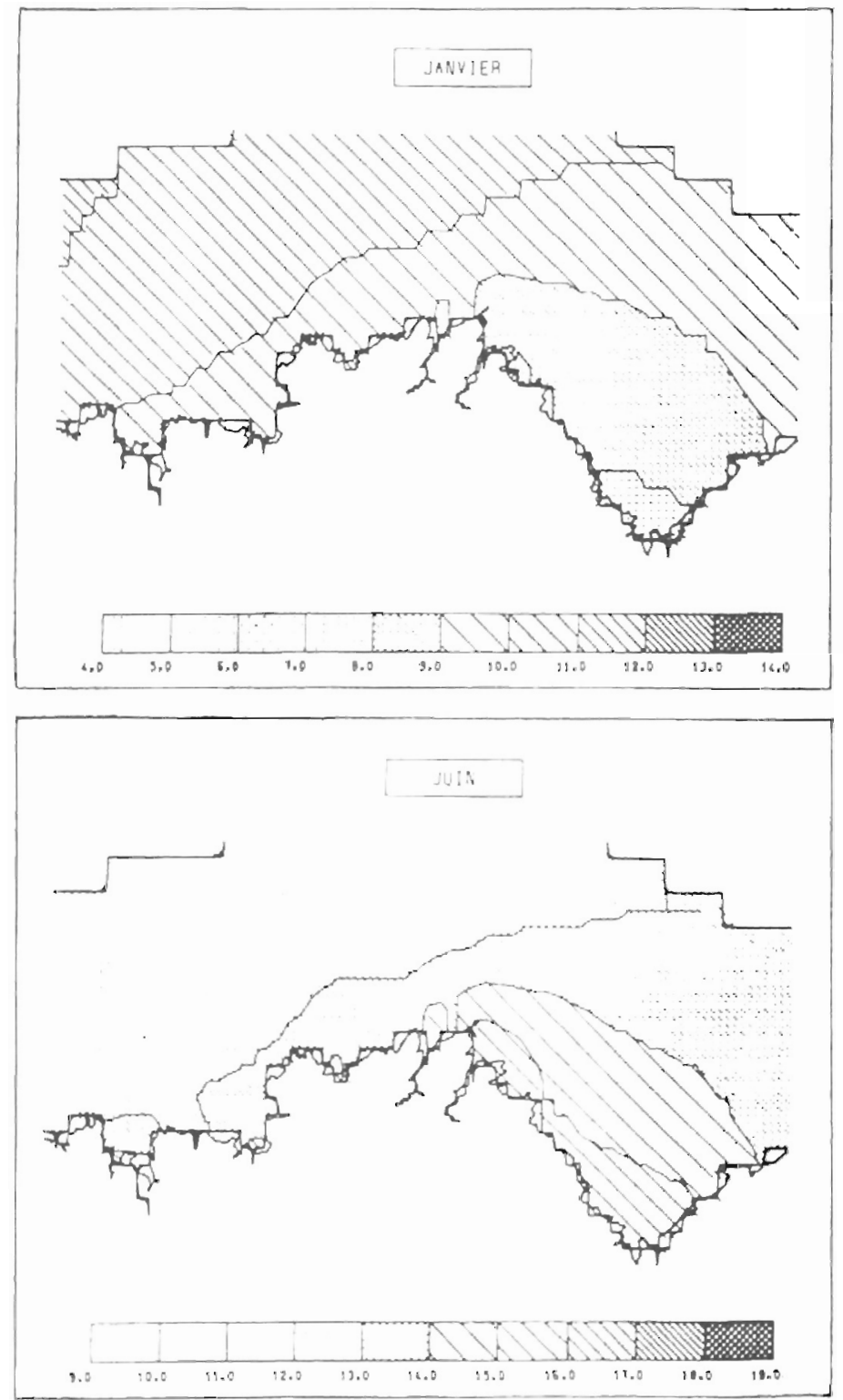

Fig. 6. Températures de surface en janvier el juin 1979.

[3] Service hydrographique et océanographique de la marine, (1968) : "Courants de marée en Manche et sur les côtes françaises de l'Atlantique", $n^{0} 550,196 \mathrm{p}$.
[4] Laboratoire National d'Hydraulique, (1987) : Catalogue sédimentologique des côtes françaises. Côtes de la Manche et de l'Atlantique. Ed. Eyrolles. 


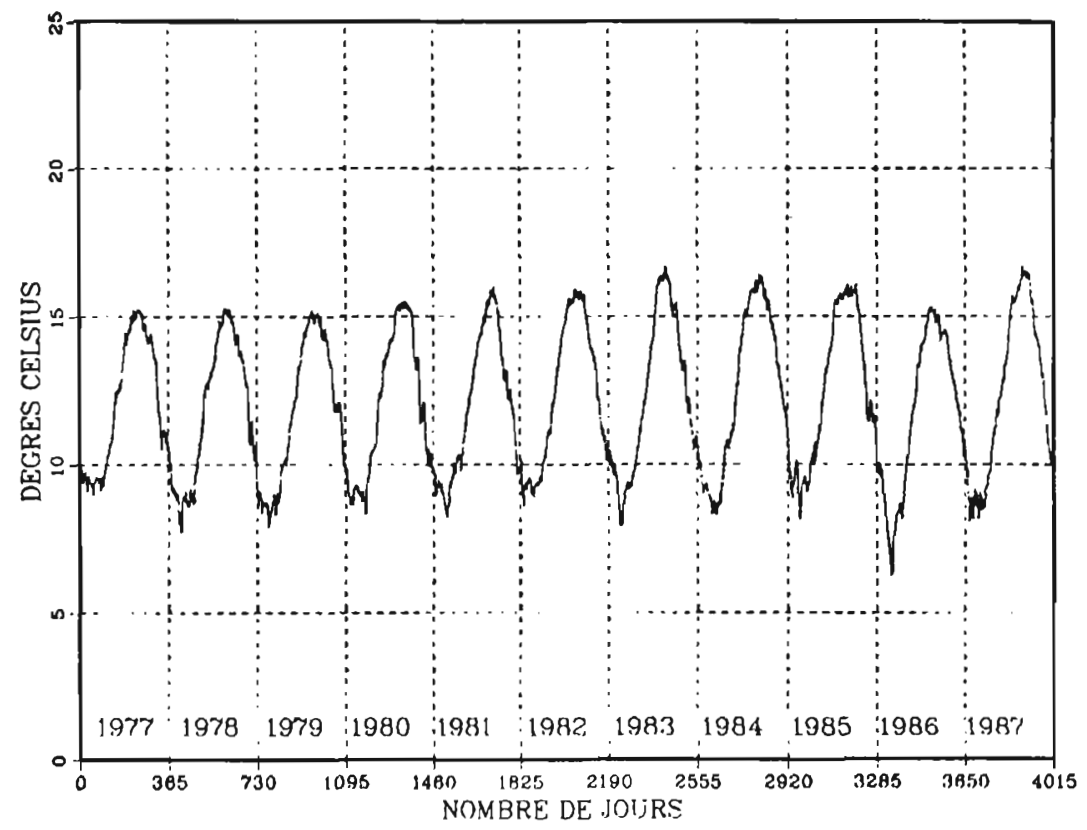

Fig. 7. Simulation de la température de surface à l'Estacade de 1977 à 1987.

[5] Klein, J.P., (1980) : Modélisation des mécanismes turbulents dans les couches marines superficielles (couche mélangée et thermocline).

Thèse de doctorat d'état, Université d'Aix-Marseille II.

[6] Niiler, P.P., et Kraus, E.B., (1977) : Modelling and prediction of the upper layers of the ocean. E.B. Kraus, Ed. Pergamon Press, p. 285.

[1] Coantic, M.F., (1978) : An introduction to turbulence in geophysics and air-sea interactions. Agardograph, $n^{\circ} 232$.

[8] Regimbaud, E., (1987) : Application de deux codes bidimensionnels en éléments finis à l'étude de l'impact thermique de centrales nucléaires. Projet de fin d'étude, école Nationale des Ponts et Chaussées.
[9] Orgby, A. (1986) : La circulation résiduelle de marée dans le golfe Normano-Breton. Thèse de doctorat, Université de Bretagne Occidentale.

[10] Prandle, D. (1978) : Monthly-mean residual flows through the Dover Strait 1949-1972. J. Mar. Bio., Ass. UK 58, 965-973.

[11] Institut scientique et technique des pêches maritimes (1976) : Température et salinité de l'eau de mer au large de Roscoff au cours des années 1959 à 1979. Trav. Stat. Biol. Roscoff, 23, 31-39.

[12] Sournia, A., Birrien, J.L., Douville, J.L., Klein, B. and Violler, M. (1987) : A daily Study of Diatom Spring Bloom at Roscoff (France) in $1985:$ I. the spring bloom within the annual cycle. Estarine, coastal and shelf science 25, 355-367. 